\title{
Electron Transparent Gold Platelets
}

\section{SUBSTRATES FOR INTERFACIAL STRUCTURAL STUDIES BY TRANSMISSION ELECTRON MICROSCOPY}

\author{
Jacobus A.A. Engelbrecht and Hendrik C. Snyman
}

Physics Department, University of Port Elizabeth, South Africa

Fundamental studies using electron microscopy in fields such as alloying and phase growth are facilitated by using thin foils of low instrinsic defect density. Gold foils or platelets produced by precipitation from aqueous solution are particularly suitable.

Interactions between different metals in contact with one another are of vital importance in many situations. It may sometimes be possible to explore the initial stages of such interactions on a microscopic scale and then to apply the knowledge gained to overcome problems which they create on the macroscopic scale. This is of particular relevance when expensive materials are involved. In this article examples of the use of the electron microscope in the study of junctions formed by the condensation from the vapour phase of other metals upon the surfaces of thin gold platelets will be highlighted. Attention will be directed mainly at three aspects of such structures in the interfacial region, namely intermetallic compound formation, flaws or defects and interfacial stresses.

When investigating bimetals by means of electron microscopy, stringent requirements are placed on the starting material or substrate that will form the basis of the bimetallic film. The requirements are that the substrate should be:

(1) very thin and clean

(2) smooth and of uniform thickness

(3) crystallographically perfect, containing few, if any, defects

(4) able to withstand high temperatures of up to $\sim 900^{\circ} \mathrm{C}$ when heated.

It is difficult to satisfy these requirements and the mostcommon technique employed for the preparation of metallic substrates has been their growth by evaporation in vacuum onto cleaved alkali halide crystals $(1,2)$ with subsequent removal by simply dissolving the alkali halide. Substrates prepared in this way suffer from three disadvantages:

(1) they contain a very high density of intrinsic defects

(2) continuous and smooth films are obtained only when they are prohibitively thick

(3) the films require subsequent manipulation and handling to remove them from the alkali halides, with possible damage to the metal film.

In the case of gold, however, substrates may advantageously be prepared by another method. Thus, a process yielding extremely thin platelets is that in which gold is precipitated from an aqueous solution of chloroauric acid by reduction with salicylic acid $(3,4)$. The resultant platelets are triangular or hexagonal in shape with the larger faces having the atomic close-packed (111) structure. Micrographs showing examples of such platelets are given in Figure 1. The crystals, which form on the top surface of the solution, are picked up on 400-mesh gold grids for examination in the electron
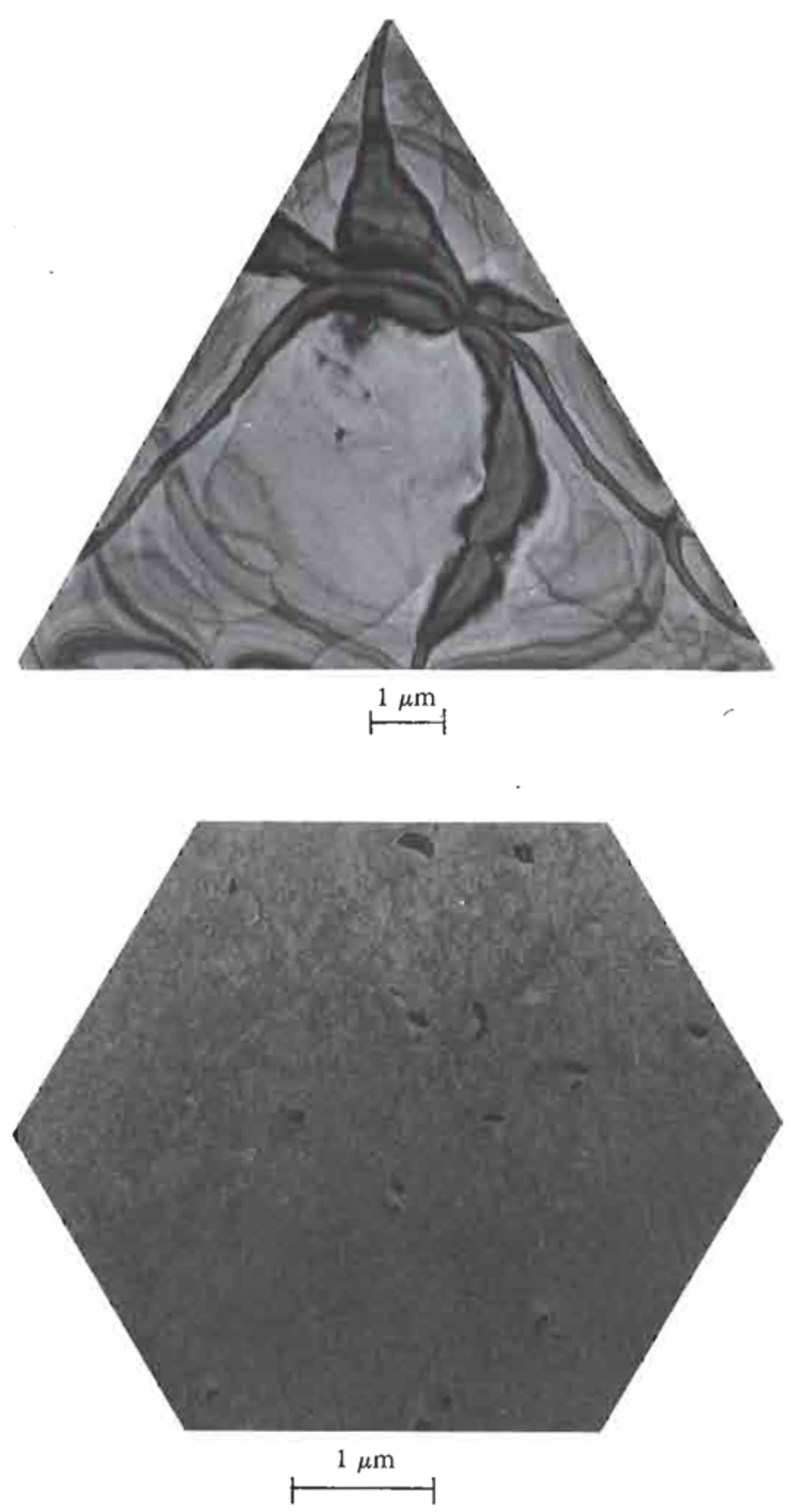

Fig. 1 Examples of triangular and hexagonal thin gold foils grown from an aqueous solution for use as substrates in electron microscopy investigations 
microscope. They are very thin, usually less than 30 nanometres in thickness, thus allowing high resolution images to be obtained in transmission electron microscopy.

Bimetal films can be prepared by vacuum-evaporation of a second metal onto such gold substrates. Normally deposition parameters such as ultimate vacuum attained, residual vacuum impurities, and the nature and orientation of the substrate are fixed. Important parameters which can be varied are the substrate temperature and overgrowth thickness. It is also possible to heat the composite layer subsequently inside the electron microscope and directly observe changes in its structure.

\section{Intermetallics}

Two interesting systems which have been investigated are the lead-gold (5) and iron-gold (6-9) junction structures. Figure 2 shows an example of a lead-gold bimetal film, as observed in the transmission electron microscope. Careful analysis of such images and their electron diffraction patterns as a function of lead thickness on the gold substrate indicates that a very thin intermetallic layer of the face centred cubic $\mathrm{Au}_{2} \mathrm{~Pb}$ Lave phase is formed during the initial stages of bimetal growth at room temperature. It is proposed that this film crystallizes after a super-saturated lead-gold solution is formed due to the diffusion of lead into the gold substrate - discussed generally by Andersson (10). Thicker layers of lead which now grow epitaxially on top of this layer have a body centred cubic structure, resulting from the two-dimensional layer or FrankVan der Merwe mode of growth (10). The normal crystal structure of lead is, in fact, face centred cubic and this study illustrates dramatically how a thin interaction layer can provide the formation upon itself of a crystal structure which would otherwise not be thermodynamically stable. Obviously the temperature at which these junctions are formed is also a critical factor, for when lead is deposited onto the gold at substrate temperatures exceeding $100^{\circ} \mathrm{C}$, another phase is observed, namely $\mathrm{AuPb}_{2}$

The results of this investigation and further studies on the stability of the intermetallics as a function of temperature are of technological significance, since lead-gold junctions are becoming prominent in semiconductor device fabrication and, in particular, in superconducting switching circuitry.

The influence of substrate temperature is even more strikingly demonstrated in the iron-gold system, Figure 3 . In the relevant experiments, the same thickness of iron was deposited on the gold platelets at different temperatures. At below $400^{\circ} \mathrm{C}$ a mixture of amorphous and body centred crystalline iron structures is formed, as well as an iron-gold interaction layer. For temperatures above $500^{\circ} \mathrm{C}$, the iron grew in the form of long needles on the gold. These needles became embedded in the gold substrates at higher temperatures. Embedding occurs as the total energy of the system is reduced

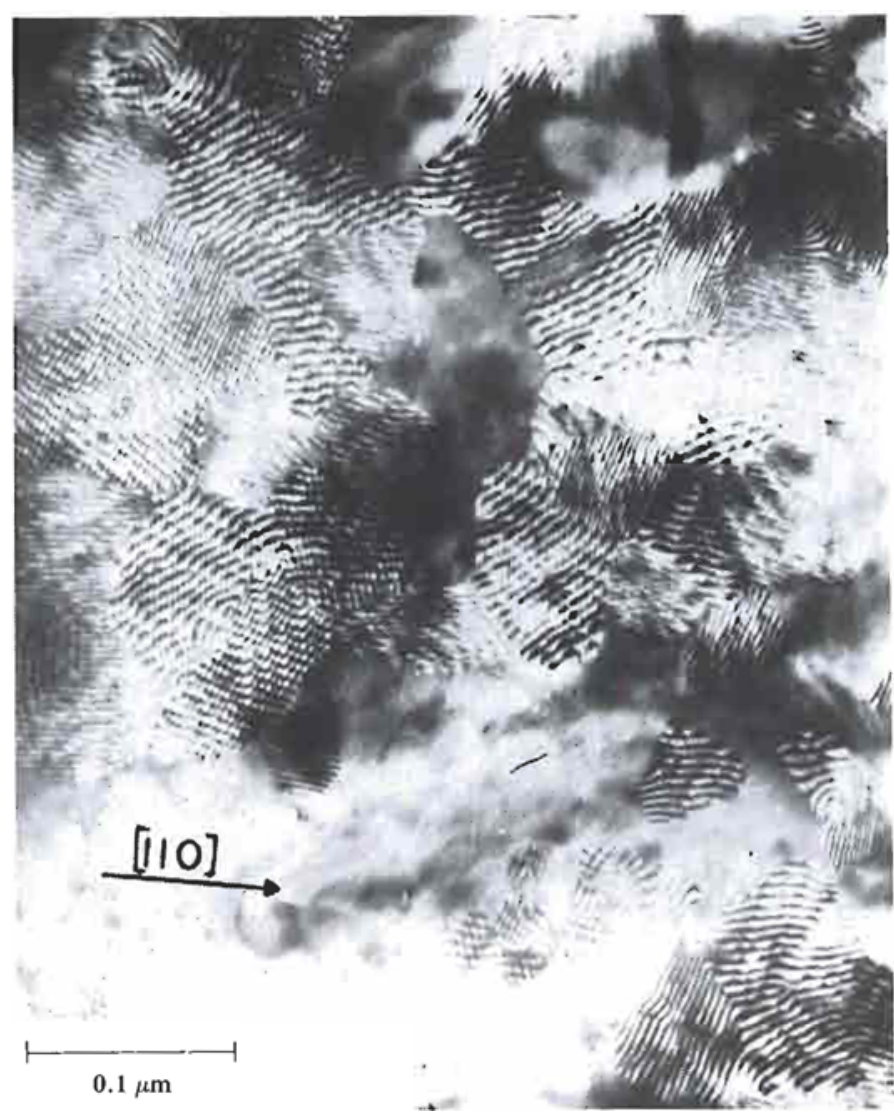

Fig. 2 Electron micrograph of a lead deposit $8 \mathrm{~nm}$ thick on gold foil. The fringe interference patterns arise from the superpositioning of the lead intermetallic matrix in various orientations on the gold matrix. In some regions no fringes are observed where the orientation of the intermetallic is out of phase with the substrate

when the needles grow into the substrate by forming the nucleation centres for the precipitation of iron from the surrounding iron-gold solid solution.

These results exemplify the important roles which both temperature and film thickness play in intermetallic compound formation.

\section{Interfacial Defects}

A unique feature of every elemental crystalline structure is that the interatomic distance between successive rows of atoms in any crystal direction is fixed. When a film of one metal grows upon the surface of another, flaws or defects, known as dislocations, may form at the interface due to mismatching or misalignment of the crystal lattices. An example of a simple dislocation is schematically shown in Figure 4.

Such interfacial defects or dislocations weaken the composite, and may have detrimental or even catastrophic consequences. In the field of micro-elecronics, for example, the many intricate interconnections that go into the making of a 


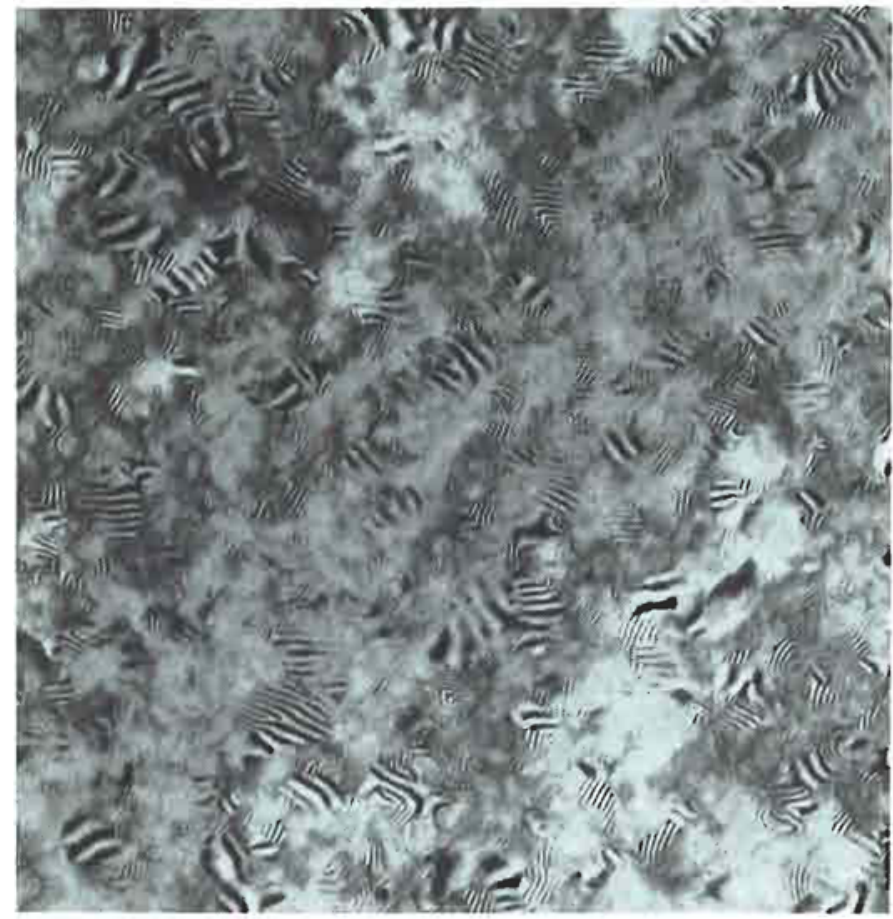

$400^{\circ} \mathrm{C}$

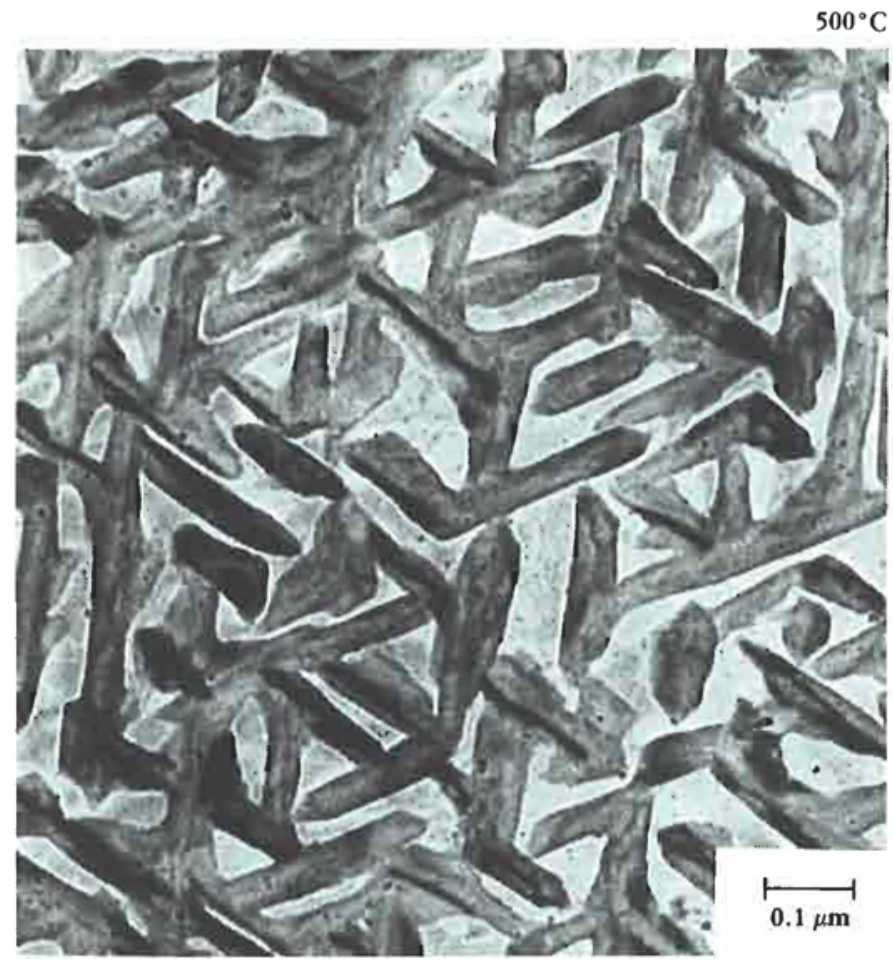

Fig. 3 Deposits of $20 \mathrm{~nm}$ thick iron on gold substrates which were held at the temperatures indicated during the growth process. A fringe pattern is observed at the lower temperature, while pure iron needles form at the higher temperature.

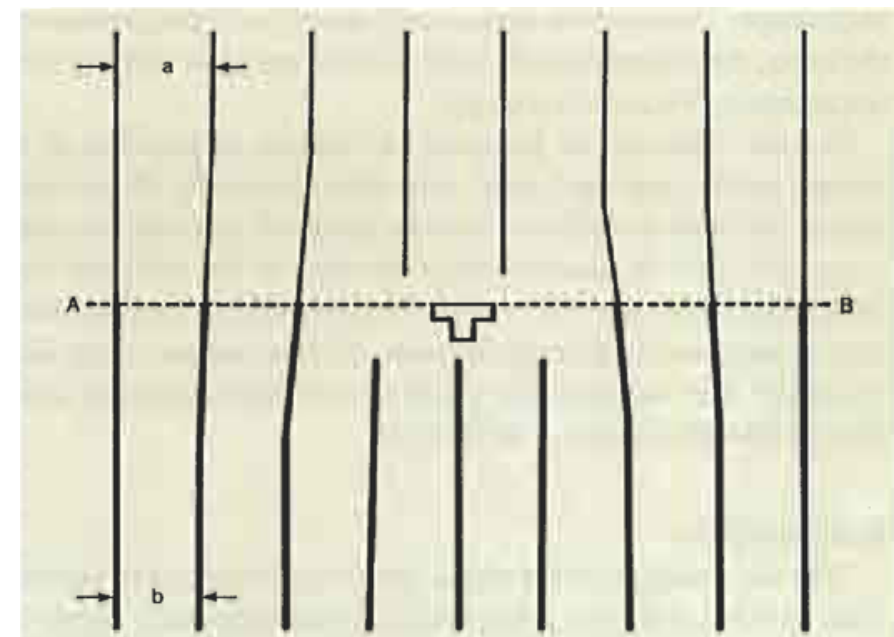

Fig.4 Schematic representation of an interfacial (edge) dislocation at interface $\mathrm{AB}$ between two materials with lattice parameters $a$ and $b$ (after Van der Merwe (11))

solid state device rely on flawless junctions or, at least, junctions where the mechanical or electrical degrading effects of crystal defects have been eliminated. This is possible only through a thorough study of the generation and elimination of interfacial defects.

In Figures 5(a) and (b) interfacial dislocations observed in the iron-silver (12) and iron-gold bimetal systems are shown. In both cases the substrate materials (silver and gold) were held at the same temperature - about $600^{\circ} \mathrm{C}$ - when the iron was deposited. Examination in the electron microscope shows that although silver and gold have the same face centred cubic structure, the only difference being a very small (less than 0.2 per cent) difference in interatomic distances, the interfacial defect structures differ dramatically. In the case of iron-silver, the dislocations lie in the interface, but with iron-gold, they are at the iron needle boundaries. Further analysis showed that while no alloying between the iron and silver occurred, the iron had in fact become embedded in the gold.

Many other bimetal systems have already been investigated and many more are being studied at present. The advantage of the gold (and silver) foils is that they are virtually defect-free at the start of any study, so that any defects observed can be ascribed solely to interaction between the materials.

\section{Stress Analysis}

No defects are formed at the interface for overgrowth thicknesses below certain critical limits up to which formation of the bimetal junction proceeds epitaxially according to the FrankVan der Merwe mode. However, one or both of the components of the bimetallic layer must undergo elastic deformation so that the rows of different atoms line up perfectly at the interface. 
Fig. 5 Typical examples of dislocations observed in thick $(>20 \mathrm{~nm})$ deposits of iron on two different substrates. In (a) iron was deposited on gold, and defects are observed on the embedded needle boundary, while in the case of iron on silver (b), the dislocations form at the interface between the iron and silver

This means that the overgrowth is under tensile or compressive stress for a comparatively thick substrate. When the thicknesses of overgrowth and substrate become comparable, however, both will be under stress, one compressive and the other tensile. Analysis of interfacial stresses is of great importance, since they may in time also cause failure of a bimetal combination. Considerable work has been devoted to the origin of these stresses (13), but few examples exist for bimetals.

Study of the silver-gold system (14) has provided some insight into internal stresses, and good agreement between theory and experiment has been obtained. Figure 6 shows a silver-gold bicrystal which has bent as a result of internal stress. An analysis of the striated pattern in the image of the triangular bicrystal due to diffraction effects provides important quantitative data regarding the distribution of the misfit stresses.

\section{Conclusion}

The use of gold foils in electron microscopy investigations in the fields of alloying, new phases and structures, stress analysis and annealing effects has proved to be ideal for fundamental studies in the cases studied so far. With the availability of the new generation of ultra high resolution analytical microscopes these substrates will become even more important for quantitative studies of interdiffusion and the atomic spatial arrangements at interfaces.

\section{References}

1 J.W. Matthews, Philos. Mag., 1966, 13, 1207-1221

2 J.W. Matthews and W.A. Jesser, Acta Metall, 1967, 15, 595-600

3 R.H. Morriss, W.R. Bottoms and R.G. Peacock, J. Appl. Phys., 1969, 39, 3016-3021

4 H.C. Snyman, F.W. Boswell and J.M. Corbett, J. Appl. Phys., 1970, 41, 816-818

5 H.C. Snyman and F.W. Boswell, Surf. Sci., 1974, 41, 21-44

6 J.A.A. Engelbrecht, J.S. Vermaak and H.C. Snyman, Thin Solid Films, $1978,48,127-132$

7 J.A.A. Engelbrecht and H.C. Snyman, Thin Solid Films, 1979, 59, 345-352

8 J.A.A. Engelbrecht and H.C. Snyman, Thin Solid Films, 1980, 69, 301-313

9 J.A.A. Engelbrecht and H.C. Snyman, Thin Solid Films, 1981, 79, 1-14

10 T.G. Andersson, Gold Bull., 1982, 15, (1), 7-18

11 J.H. Van der Merwe, 'Single Crystal Films' edited by M.H. Francombe and H. Sato, Pergamon Press, N.Y., 1964, p. 139

12 H.C. Snyman and G.H. Olsen, J. Appl. Phys, , 1973, 44, 889-891

13 R.W. Hoffman, 'The Usc of Thin Films in Physical Investigations', Academic Press, N.Y., 1966

14 H.C. Snyman and F.W. Boswell, Surf. Sci, 1973, 36, 74-80

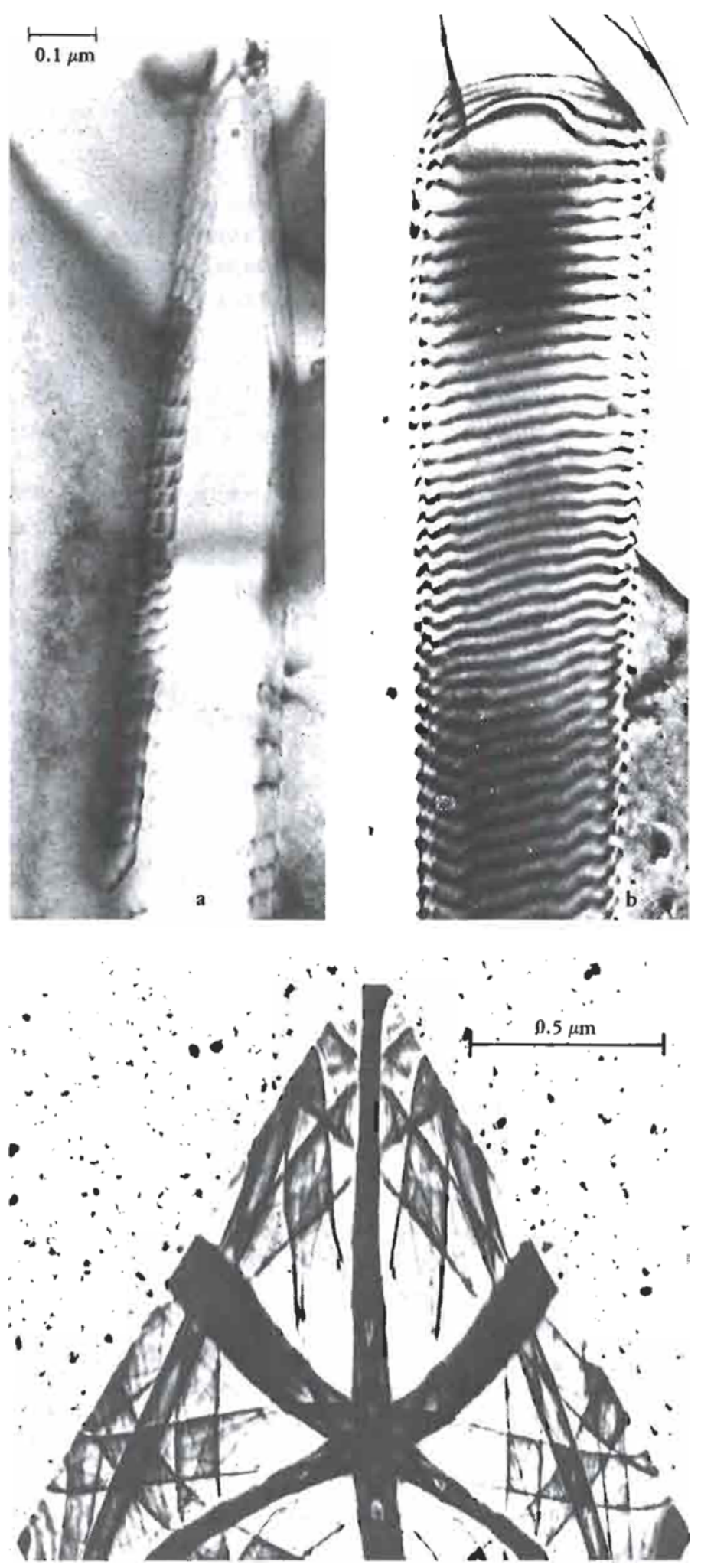

Fig. 6 An electron micrograph showing the bend contours in a silver-gold bimetal film. (The dark spots in the background are due to the silver on the carbon carrier film) 